\title{
Childhood Obesity Is Associated with Changes in the Serum Metabolite Profile
}

\author{
Simone Wahl ${ }^{a}$ Zhonghao $\mathrm{Yu}^{\mathrm{a}}$ Michaela Kleber ${ }^{\mathrm{b}}$ Paula Singmann ${ }^{\mathrm{a}}$ \\ Christina Holzapfel $^{a, c}$ Ying He ${ }^{a}$ Kirstin Mittelstrass ${ }^{a}$ Alexey Polonikov ${ }^{d}$ \\ Cornelia Prehn $^{\text {e }}$ Werner Römisch-Margl ${ }^{f}$ Jerzy Adamski ${ }^{e}$ Karsten Suhre ${ }^{f}$ \\ Harald Grallert ${ }^{a}$ Thomas Illig ${ }^{a}$ g Rui Wang-Sattler ${ }^{a}$ Thomas Reinehr ${ }^{b}$ \\ a Research Unit of Molecular Epidemiology, Helmholtz Zentrum München, German Research \\ Center for Environmental Health, Neuherberg, bVestische Kinder- und Jugendklinik Datteln, \\ University of Witten-Herdecke, Datteln, ${ }^{C}$ Else Kröner-Fresenius-Center of Nutritional Medicine, \\ Technische Universität München, Freising, ${ }^{\mathrm{d}}$ Department of Biology, Medical Genetics and \\ Ecology, Kursk State Medical University, Kursk, Russia, e Institute of Experimental Genetics, \\ Genome Analysis Center, Helmholtz Zentrum München, German Research Center for

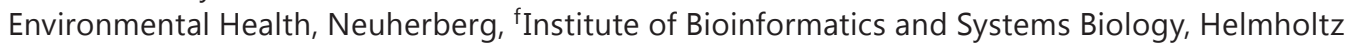 \\ Zentrum München, German Research Center for Environmental Health, Neuherberg, ${ }^{9}$ Medical \\ School Hanover, Hannover Unified Biobank, Hanover, Germany
}

\section{Key Words}

BMI • Childhood obesity $\cdot$ Lipid metabolism • Metabolite profile $\cdot$ Serum $\cdot$ Biomarker $•$ Metabolomics

\begin{abstract}
Objective: The human serum metabolite profile is reflective of metabolic processes, including pathophysiological changes characteristic of diseases. Therefore, investigation of serum metabolite concentrations in obese children might give new insights into biological mechanisms associated with childhood obesity. Methods: Serum samples of 80 obese and 40 normalweight children between 6 and 15 years of age were analyzed using a mass spectrometrybased metabolomics approach targeting 163 metabolites. Metabolite concentrations and metabolite ratios were compared between obese and normal-weight children as well as between children of different pubertal stages. Results: Metabolite concentration profiles of obese children could be distinguished from those of normal-weight children. After correction for multiple testing, we observed 14 metabolites (glutamine, methionine, proline, nine phospholipids, and two acylcarnitines, $\left.p<3.8 \times 10^{-4}\right)$ and 69 metabolite ratios $\left(p<6.0 \times 10^{-6}\right)$ to be significantly altered in obese children. The identified metabolite markers are indicative of oxidative stress and of changes in sphingomyelin metabolism, in $\beta$-oxidation, and in pathways associated with energy expenditure. In contrast, pubertal stage was not associated with
\end{abstract}


metabolite concentration differences. Conclusion: Our study shows that childhood obesity influences the composition of the serum metabolome. If replicated in larger studies, the altered metabolites might be considered as potential biomarkers in the generation of new hypotheses on the biological mechanisms behind obesity.

Copyright (C 2012 S. Karger GmbH, Freiburg

\section{Introduction}

The prevalence of childhood obesity has been increasing dramatically worldwide over the past three decades [1]. The underlying cause is a complex interaction between predisposing genetic factors and the change of environmental factors such as nutritional habits $[2$, 3]. This trend is of great concern, considering the manifold consequences of obesity on children's health. These include metabolic disturbances such as insulin resistance, dyslipidemia, and hypertension, which in turn promote the development of cardiovascular diseases in childhood as well as in later life $[2,4]$.

For the development of appropriate treatment strategies, a good understanding of obesity-related pathophysiological mechanisms is inevitable. In recent years, advanced laboratory techniques and data processing tools have been developed, allowing for largescale, simultaneous investigation of a large number of metabolites in human biofluids or tissues [5-7]. The human serum metabolite profile is reflective of metabolic processes including disease-related changes [8]. Therefore, investigation of serum metabolite concentrations in obese children might give new insights into biological mechanism associated with childhood obesity. Few studies have already examined obesity-associated changes in the serum metabolome in adults [9-12] and adolescents [13].

In the present study, we applied a targeted metabolomics approach to identify serum metabolites associated with childhood obesity. The foremost aims of this study were i) to investigate whether the concentration profile of serum metabolites differed between obese and normal-weight children, ii) to identify potential obesity-associated metabolite biomarkers, and iii) to analyze the association of pubertal stage with the metabolite profile.

\section{Material and Methods}

Study Population and Anthropometric Measures

In the years 2008-2009, 80 obese children and adolescents aged 6-15 years from the outpatient clinic for obesity in the Vestische Kinder- und Jugendklinik Datteln, Germany, and 40 normal-weight children with a similar age and sex distribution were examined. All children were born in Germany. Children with syndromal obesity as well as psychiatric or endocrine disorders including type 2 diabetes mellitus were excluded. The Ethics Committee of the University of Witten/Herdecke approved this study. Written informed consent was obtained from all subjects and their parents.

Height was measured to the nearest centimeter using a rigid stadiometer. Undressed weight was measured to the nearest $0.1 \mathrm{~kg}$ using a calibrated balance scale. Obesity was defined as BMI above the 97th percentile of German population-specific data [14]. All obese children met obesity criteria of the International Task Force of Obesity, being above the population-, age-, and sex-specific percentile translating to a BMI of $30 \mathrm{~kg} / \mathrm{m}^{2}$ at the age of 18 [15]. BMI percentiles and standard deviation scores (SDS-BMI) were calculated based on Cole's LMS method [16]. Pubertal stage was assessed according to Marshall and Tanner [17, 18] and categorized into three stages based on pubic hair and genital stages: prepubertal = boys/girls with pubic hair stage I and gonadal/breast stage I, pubertal = boys/girls with pubic hair stage $\geq$ II and gonadal/ breast stage $\geq$ II and late/postpubertal = boys with change of voice and girls with menarche. 
Wahl et al.: Childhood Obesity Is Associated with Changes in the Serum Metabolite Profile

\section{Sampling and Metabolite Measurement}

Blood samples were taken at 8 a.m. after overnight fasting for at least $10 \mathrm{~h}$. Following coagulation at room temperature, blood samples were centrifuged for $10 \mathrm{~min}$ at $8,000 \mathrm{rpm}$. Aliquoted serum samples were stored at $-80{ }^{\circ} \mathrm{C}$ and thawed at room temperature for the metabolomics assay.

We used the metabolomics AbsoluteIDQ ${ }^{\mathrm{TM}}$ kit p150 (Biocrates Life Sciences AG, Innsbruck, Austria) for the quantification of 163 metabolites, following the instructions described in the manufacturer's manual. Liquid handling of serum samples was performed with a Hamilton Micro Lab Star robot (Hamilton Bonaduz AG, Bonaduz, Switzerland). Samples were analyzed on an API4000 LC/MS/MS System (AB Sciex Deutschland GmbH, Darmstadt, Germany) equipped with an HTC PAL autosampler (CTC Analytics, Zwingen, Switzerland) and an electrospray ionization (ESI) source which was used in both positive and negative mode. MS/MS analysis was run in Multiple Reaction Monitoring (MRM) mode. The entire analytical process was controlled by the Analyst 1.4 software and the MetIQ ${ }^{\mathrm{TM}}$ soft ware package, the latter being an integral part of the AbsoluteIDQ kit. Metabolite concentrations were determined with the MetIQ software.

Measurements took place in two separate batches. For data quality assessment, 43 of the samples of the first batch were remeasured in the second one. Also, a reference sample was measured five times on each of the two batches. We excluded metabolites from the analysis that failed to meet two or more of the following quality criteria:

i) The concentration of the metabolite should be above the limit of detection (LOD) specified by the manufacturer in at least $60 \%$ of the samples.

ii) The Pearson's correlation coefficient of the metabolite concentrations in the 43 repeatedly measured samples should be at least 0.5 between the two batches.

iii) For each batch, the coefficient of variation (CV) for the metabolite concentration in the reference samples should not be higher than 0.2 .

In total, 130 metabolites passed the quality control. Most of the 33 excluded metabolites were characterized by concentrations below or marginally above the LOD. As a consequence, measurement stability was assumably affected in these cases. In accordance with the kit instruction all measurements were multiplied by a metabolite- and batch-specific correction factor to further correct for a potential batch effect.

The metabolite spectrum targeted by the used kit is shown in supplementary table 1 (see supplementary material). The 130 metabolites included in our analysis comprised 24 conjugated carnitines (acylcarnitines (Cx:y), hydroxylacylcarnitines (C(OH)x:y) and dicarboxylacylcarnitines (Cx:y-DC), free carnitine (CO), 14 amino acids, hexose (H1), 32 diacyl phosphatidylcholines (PC aa Cx:y), 35 acyl-alkyl phosphatidylcholines (PC ae Cx:y), 9 lysophosphatidylcholines (LPC a Cx:y) as well as 14 sphingomyelins (SM Cx:y) and hydroxysphingomyelins (SM (OH) Cx:y), where Cx:y abbreviates the lipid side chain composition, $\mathrm{x}$ and $\mathrm{y}$ denoting the number of carbons and double bonds, respectively. As a point to note, the analytical technique applied here is not capable of determining the precise position of the double bonds and - in the case of PCs - the distribution of carbon atoms between the two fatty acid side chains. We report all metabolite concentrations in $\mu \mathrm{mol} / \mathrm{l}$. In the following, we refer to metabolite concentrations simply as metabolites, and to metabolite concentration ratios as metabolite ratios.

\section{Statistical Analysis}

All calculations were performed in the statistical environment $R$, version 2.10 .1 [19]. Anthropometric characteristics of obese and normal-weight children were compared using Wilcoxon signed-rank and Pearson's chi-square tests.

To examine the degree to which the serum metabolite profiles of obese and normal-weight children and of children of different pubertal stages were separated, Partial Least Squares (PLS) analysis with 10 -fold cross-validation was conducted on the scaled and centered matrix of metabolite concentrations using the $R$ package $p l s$ [20]. Data were visualized by plotting the cross-validated scores of the first two components in a scores plot where each point represents an individual serum sample.

To determine whether single metabolite concentrations differed between obese and normal-weight children, we performed logistic regression analyses with weight status (obese vs. normal-weight) as the dependent variable and the natural logarithm of single metabolite concentrations and the covariates age, sex, and pubertal stage as the independent variables. A similar analysis was performed to investigate associations between pubertal stage (prepubertal vs. pubertal/postpubertal) and metabolite concentrations, adjusted for age and sex and for sex only. 
Table 1. Anthropometric characteristics of normal-weight and obese children ${ }^{\mathrm{a}}$

\begin{tabular}{llll}
\hline Parameter & $\begin{array}{l}\text { Normal-weight } \\
(\mathrm{n}=40)\end{array}$ & $\begin{array}{l}\text { Obese } \\
(\mathrm{n}=80)\end{array}$ & p value \\
\hline Age, years & $11.1 \pm 2.8$ & $10.9 \pm 2.1$ & 0.700 \\
Sex (\% male/female) & $62.5 / 37.5$ & $52.5 / 47.5$ & 0.338 \\
Pubertal stage (\% prepubertal/pubertal/postpubertal) & $47.5 / 35.0 / 17.5$ & $51.3 / 36.3 / 12.5$ & 0.706 \\
Weight, kg & $36.5 \pm 14.2$ & $65.2 \pm 17.6$ & $<0.001$ \\
BMI, kg/m $^{2}$ & $17.2 \pm 2.1$ & $27.7 \pm 4.0$ & $<0.001$ \\
SDS-BMI & $-0.4 \pm 0.8$ & $2.4 \pm 0.4$ & $<0.001$ \\
\hline
\end{tabular}

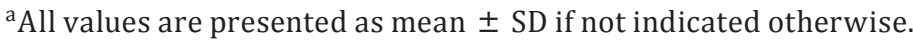

${ }^{\mathrm{b}}$ Standard deviation score of BMI.

In a subsequent step, we repeated the analysis for obesity with ratios of all possible pairs of metabolites, allowing for the discovery of differences between obese and normal-weight children that are otherwise masked by a large intra-group variability in the single metabolite concentrations [21, 22]. In addition, changes in metabolite ratios might reflect biological situations such as changes in enzyme activities or imbalances in metabolic pathways. For all regression analyses, we considered an experiment-wise type I error of 0.05 as statistically significant. Correction for multiple testing using the Bonferroni correction resulted in a significance level of $p=0.05 / 130=3.8 \times 10^{-4}$ and $p=0.05 / 8385=6.0 \times 10^{-6}$ for single metabolites and metabolite ratios, respectively. In the case of metabolite ratios, we calculated the p gain, defined as the minimum of the two $p$ values for the single metabolite analyses divided by the $p$ value for the metabolite ratio [21]. The higher the $\mathrm{p}$ gain for a metabolite ratio, the more a low $\mathrm{p}$ value for the metabolite ratio is due to a change in the ratio rather than to a strong change in one of the single metabolites alone. In this study, we focused on metabolite ratios with a gain of at least $1.0 \times 10^{3}$.

\section{Results}

\section{Characteristics of the Study Population}

Anthropometric characteristics of the children are presented in table 1 . Obese and normal-weight children did not differ significantly in terms of sex, age, and pubertal stage distribution. By design, body weight, BMI, and SDS-BMI were significantly higher in the group of obese children $(\mathrm{p}<0.001)$.

\section{The Serum Metabolite Profile in Obesity}

The associations of obesity and pubertal stage with the metabolite profile were first investigated by means of PLS analysis. We observed a distinct separation of obese and normal-weight children by the first two components, which accounted for $22.1 \%$ and $29.7 \%$ of the total variance in the metabolite data, respectively (fig. 1). In contrast, we could not observe a separation of children of different pubertal stages.

After correction for multiple testing, concentrations of 14 metabolites differed significantly between obese and normal-weight children (table 2). Concentrations of two acylcarnitines (C12:1 and C16:1) were significantly increased in obese compared to normal-weight children, while concentrations of the remaining 12 metabolites (glutamine, methionine, proline, PC ae C34:1, C34:2, C34:3, C36:2, C36:3 and C38:2, LPC a C18:1, C18:2 and C20:4) were significantly decreased in obese children. Logistic regression results for all 130 metabolites are shown in supplementary table 2 (see supplementary material). Performing a similar analysis for pubertal stage, we did not identify any significant associations with metabolite concentrations, neither with nor without adjustment for age (data not shown). 
Table 2. Metabolites with concentrations differing significantly between normal-weight and obese children

\begin{tabular}{|c|c|c|c|c|c|}
\hline \multirow{2}{*}{$\begin{array}{l}\text { Metabolite } \\
\text { Acylcarnitines }\end{array}$} & \multirow[t]{2}{*}{$\begin{array}{l}\text { Normal-weight ( } \mathrm{n} \\
=40)^{\mathrm{a}}\end{array}$} & \multirow[t]{2}{*}{ Obese $(n=80)^{a}$} & \multicolumn{2}{|c|}{ 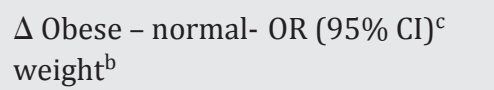 } & \multirow[t]{2}{*}{$\mathrm{p}$ value } \\
\hline & & & & & \\
\hline C12:1 & $0.10 \pm 0.03$ & $0.13 \pm 0.04$ & $32.3 \%$ & $3.62(2.09,6.98)$ & $2.6 \times 10^{-5}$ \\
\hline C16:1 & $0.03 \pm 0.01$ & $0.04 \pm 0.01$ & $20.8 \%$ & $2.74(1.64,4.98)$ & $3.3 \times 10^{-4}$ \\
\hline \multicolumn{6}{|l|}{ Amino acids } \\
\hline Glutamine & $638.62 \pm 109.94$ & $509.43 \pm 114.91$ & $-20.2 \%$ & $0.20(0.09,0.38)$ & $7.0 \times 10^{-6}$ \\
\hline Methionine & $35.09 \pm 8.76$ & $27.55 \pm 6.81$ & $-21.5 \%$ & $0.28(0.15,0.49)$ & $3.4 \times 10^{-5}$ \\
\hline Proline & $258.91 \pm 91$ & $180.88 \pm 63.23$ & $-30.1 \%$ & $0.27(0.14,0.46)$ & $1.3 \times 10^{-5}$ \\
\hline \multicolumn{6}{|c|}{ Acyl-alkyl phosphatidylcholines } \\
\hline PC ae C34:1 & $10.26 \pm 3.20$ & $8.26 \pm 2.07$ & $-19.5 \%$ & $0.34(0.18,0.59)$ & $3.0 \times 10^{-4}$ \\
\hline PC ae C $34: 2$ & $12.70 \pm 4.07$ & $9.32 \pm 2.69$ & $-26.6 \%$ & $0.29(0.15,0.50)$ & $4.1 \times 10^{-5}$ \\
\hline PC ae $\mathrm{C} 34: 3$ & $9.22 \pm 3.31$ & $6.95 \pm 2.02$ & $-24.6 \%$ & $0.39(0.23,0.62)$ & $2.3 \times 10^{-4}$ \\
\hline PC ae C36:2 & $13.63 \pm 3.69$ & $10.25 \pm 2.55$ & $-24.8 \%$ & $0.24(0.12,0.43)$ & $1.1 \times 10^{-5}$ \\
\hline PC ae C36:3 & $8.51 \pm 2.52$ & $6.61 \pm 1.86$ & $-22.2 \%$ & $0.33(0.17,0.56)$ & $2.0 \times 10^{-4}$ \\
\hline PC ae C38:2 & $1.96 \pm 0.51$ & $1.55 \pm 0.48$ & $-21.0 \%$ & $0.36(0.20,0.59)$ & $1.8 \times 10^{-4}$ \\
\hline \multicolumn{6}{|c|}{ Lysophosphatidylcholines } \\
\hline LPC a C18:1 & $17.13 \pm 4.59$ & $12.73 \pm 3.96$ & $-25.7 \%$ & $0.29(0.15,0.49)$ & $1.8 \times 10^{-5}$ \\
\hline LPC a C18:2 & $36.37 \pm 11.44$ & $18.09 \pm 5.07$ & $-50.3 \%$ & $0.05(0.01,0.13)$ & $5.7 \times 10^{-8}$ \\
\hline LPC a C20:4 & $5.82 \pm 1.77$ & $4.4 \pm 1.21$ & $-24.4 \%$ & $0.36(0.20,0.58)$ & $1.2 \times 10^{-4}$ \\
\hline
\end{tabular}

Cx:y = acyl-group with chain length $\mathrm{x}$ and $\mathrm{y}$ double bonds; PC ae = acyl-alkyl phosphatidylcholine; LPC a = lysophosphatidylcholine with acyl chain.

${ }^{a}$ All values are presented as mean \pm SD of serum metabolite concentration in $\mu \mathrm{mol} / \mathrm{l}$.

${ }^{b}$ Percentage $(\%)$ difference between values for obese and normal-weight children.

${ }^{\mathrm{c}} \mathrm{OR}$ of logistic regression analysis with respective $95 \% \mathrm{CI}$; age, sex and pubertal stage were included as covariates; only metabolites whose concentrations differed significantly after adjustment for multiple testing $\left(\mathrm{p} \leq 3.8 \times 10^{-4}\right)$ were included.

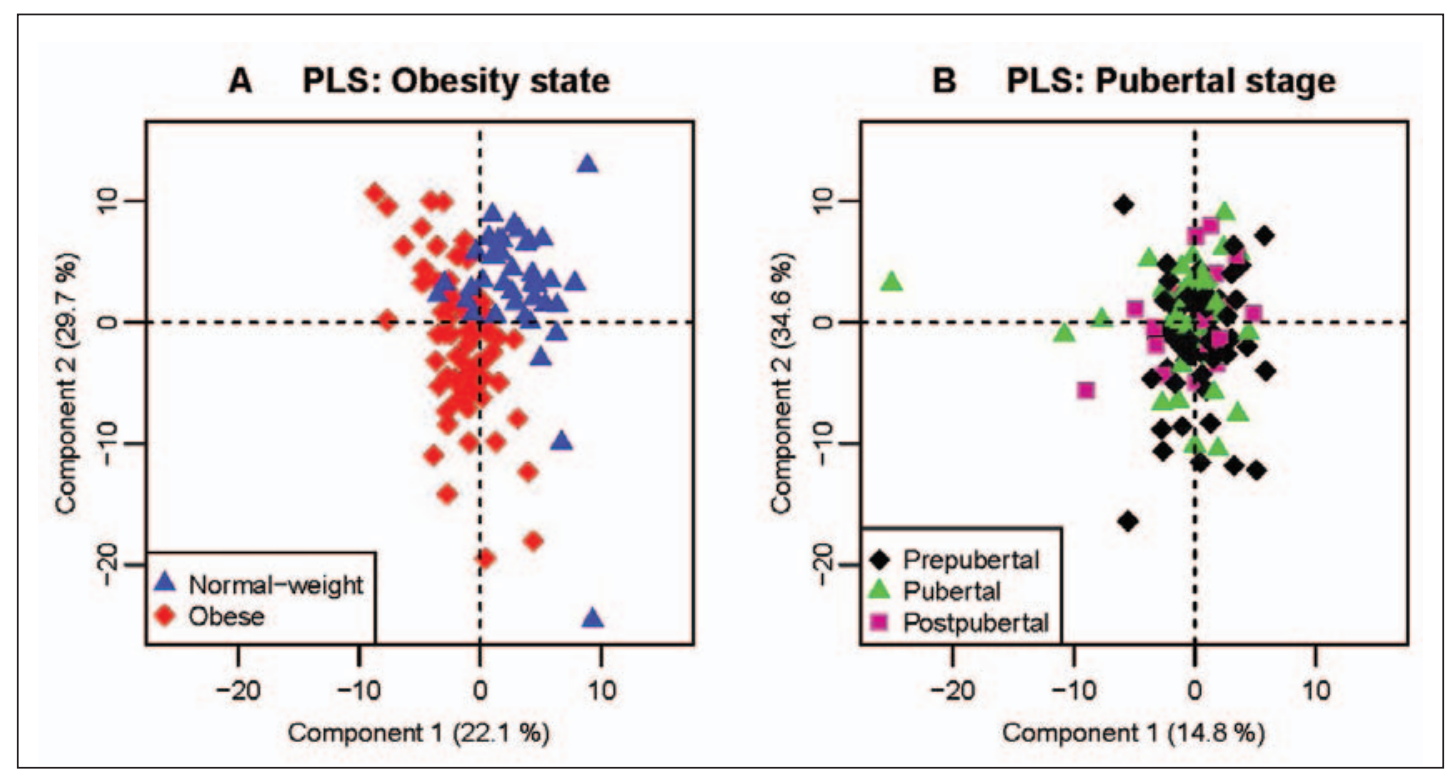

Fig. 1. PLS analysis results. Cross-validated scores plot of the first two components. Outcome was A, obesity, and B, pubertal stage. Percentages in parentheses represent the \% variance in the metabolite data accounted for by the respective component. 
Table 3. Selection of metabolite concentration ratios differing significantly between normal-weight and obese children

\begin{tabular}{lccc}
\hline Metabolite ratio & OR $(95 \% \mathrm{CI})^{\mathrm{a}}$ & p value & p gain \\
\hline \multicolumn{2}{l}{ Saturated / unsaturated lysophosphatidylcholine ratios } & & \\
LPC a C16:0 / LPC a C20:3 & $6.0(3.3,12.2)$ & $6.3 \times 10^{-8}$ & $1.3 \times 10^{5}$ \\
LPC a C18:0 / LPC a C20:3 & $9.6(4.5,24.6)$ & $1.3 \times 10^{-7}$ & $6.1 \times 10^{4}$ \\
LPC a C16:0 / LPC a C20:4 & $6.5(3.5,13.8)$ & $1.0 \times 10^{-7}$ & $1.1 \times 10^{3}$ \\
LPC a C18:0 / LPC a C20:4 & $8.4(4.1,20.1)$ & $1.1 \times 10^{-7}$ & $1.1 \times 10^{3}$ \\
Lysophosphatidylcholine / phosphatidylcholine ratios & & \\
LPC a C18:0 / PC aa C36:2 & $8.3(4.2,19.6)$ & $6.5 \times 10^{-8}$ & $1.2 \times 10^{5}$ \\
LPC a C18:0 / PC aa C36:1 & $5.7(3.1,12.0)$ & $4.4 \times 10^{-7}$ & $2.0 \times 10^{4}$ \\
LPC a C16:0 / PC aa C32:0 & $5.3(2.9,10.7)$ & $3.3 \times 10^{-7}$ & $2.5 \times 10^{3}$ \\
LPC a C16:0 / PC aa C36:2 & $3.8(2.2,6.9)$ & $3.3 \times 10^{-6}$ & $2.3 \times 10^{3}$ \\
Sphingomyelin / phosphatidylcholine ratios & & & \\
SM (OH) C22:1 / PC ae C38:4 & $5.1(2.9,10.3)$ & $4.8 \times 10^{-7}$ & $1.1 \times 10^{4}$ \\
SM C24:0 / PC aa C36:2 & $4.2(2.4,7.8)$ & $1.3 \times 10^{-6}$ & $6.0 \times 10^{3}$ \\
SM C16:1 / PC ae C32:1 & $3.7(2.2,6.7)$ & $3.2 \times 10^{-6}$ & $4.9 \times 10^{3}$ \\
SM C18:1 / PC ae C38:4 & $5.8(3.0,13.0)$ & $1.6 \times 10^{-6}$ & $3.2 \times 10^{3}$ \\
\hline
\end{tabular}

Cx:y = acyl-group with chain length $\mathrm{x}$ and y double bonds; LPC a = lysophosphatidylcholine with acyl chain; PC aa = diacyl phosphatidylcholine; $\mathrm{PC}$ ae = acyl-alkyl phosphatidylcholine; SM = sphingomyelin; SM $(\mathrm{OH})=$ hydroxysphingomyelin.

${ }^{\mathrm{a}} \mathrm{OR}$ of logistic regression analysis with respective $95 \% \mathrm{CI}$; age, sex and pubertal stage were included as covariates; only metabolites pairs whose concentration ratios differed significantly after adjustment for multiple testing $\left(\mathrm{p} \leq 6.0 \times 10^{-6}\right)$ and had a p-gain $\geq 1.0 \times 10^{3}$ were included in the table.

\section{Metabolite Ratios}

Comparing metabolite ratios between obese and normal-weight children in logistic regression analyses revealed significant differences in 69 ratios after correction for multiple testing $\left(\mathrm{p} \leq 6.0 \times 10^{-6}\right)$ and applying a p gain cutoff at $\geq 1.0 \times 10^{3}$ (supplementary table 3 ; see supplementary material). Results for selected representative ratios are shown in table 3 . Ratios between saturated and unsaturated LPCs, between saturated LPCs and PCs and between SMs and PCs were increased in obese compared to normal-weight children. As an example, the odds ratio for being in the obese group of the LPC a C18:0 / LPC a C20:4 ratio was $8.4(4.1,20.1)$ with a $p$ value of $1.1 \times 10^{-7}$ and a pain of $1.1 \times 10^{3}$.

\section{Discussion}

This study compared serum metabolite concentrations of obese and normal-weight children in a systematic metabolomics approach. 14 metabolites and 69 metabolite ratios were significantly different in obese compared to normal-weight children.

\section{Metabolite Changes Associated with Obesity}

The observed higher concentrations of the acylcarnitines C12:1 and C16:1 in obese compared to normal-weight children are consistent with findings in adults. In a study comparing acylcarnitine species of 12 lean and 14 obese adults, serum concentrations of several medium- to long-chain acylcarnitines were increased in the obese compared to the non-obese participants [9]. Acylcarnitines are formed from acyl-coenzyme A (CoA) by the outer mitochondrial enzyme carnitine palmitoyltransferase 1 (CPT1) as a prerequisite to 
acyl transport into mitochondria where $\beta$-oxidation takes place [23]. Elevated free fatty acid (FFA) concentrations, as have been observed in the serum of obese children [24], may exceed the capacity of $\beta$-oxidation and downstream pathways such as the mitochondrial electron transport chain, the activities of which have been shown to be reduced in obese subjects $[25,26]$. As a consequence, incompletely oxidized fatty acid products such as acylcarnitines might accumulate, exit the cell, and are found increased in the blood [9, 27]. A recent study in obese and normal-weight adolescents reported unchanged longer-chain acylcarnitines and decreased concentrations of medium- to short-chain acylcarnitine species [13]. They explain the inconsistency with findings in adults with the existence of adaptive mitochondrial mechanisms in youth. Our results do not support such a hypothesis.

We found significantly reduced glutamine, methionine and proline concentrations in obese compared to normal-weight children. Thus, we replicated the findings of an earlier study reporting reduced levels of methionine and proline in extremely obese children [28]. To our knowledge, no study on obese children has shown a reduction of glutamine. However, findings of other studies point towards an obesity-associated activation of the hexosamine pathway [29-31]. Glucosamine and hexosamines are known to contribute to the development of insulin resistance $[29,32]$. In addition, activation of the hexosamine pathway reduces energy expenditure [30].

Our study confirmed that acyl-alkyl PC concentrations are significantly lower in the obese state [10]. A subclass of acyl-alkyl PCs are the so-called plasmalogens which have antioxidant properties [33]. The observed decrease in acyl-alkyl PCs in obese children may reflect a consumption of plasmalogens during oxidative stress, which is known to play a role in childhood obesity [34].

We observed significantly increased ratios between saturated LPCs (C16:0, C18:0) and PCs in obese children. Saturated LPC concentrations were also marginally increased. LPCs are derived from PCs during LDL oxidation via either the lecithin-cholesterol acyltransferase (LCAT) or the lipoprotein-associated phospholipase A2 (LpPLA2) pathway [35]. In fact, LpPLA2 activity has been reported to be increased in obese children [36]. As a major component of oxidized LDL, saturated LPCs exert pro-atherogenic and pro-inflammatory effects and impair insulin signaling [35, 37].

Obese children demonstrated significantly decreased concentrations of the unsaturated LPCs C18:1, C18:2 and C20:4. Furthermore, ratios between saturated and unsaturated LPCs were significantly increased. Our findings are in line with a recent metabolomics study in overweight/obese and normal-weight men showing significantly reduced concentrations of LPC C18:1 and increased concentrations of LPC C18:0 along with a reduced ratio of dietary polyunsaturated to saturated fatty acid intake [12]. The arising assumption that fatty acid composition of serum (lyso-)phospholipids partly reflects an individual's medium-term dietary fatty acid intake is supported by the reported correlations between dietary and plasma phospholipid fatty acids [38, 39]. So far, there is little evidence for a role of specific dietary characteristics, including fatty acid balance, in childhood obesity risk [40].

Our observation that SM/PC ratios were significantly increased in obese children may reflect an increased rate of the sphingomyelin synthase (SMS) reaction [41]. We did not observe a significant increase in single SM concentrations. This may depict the equilibrium of SM synthesis and metabolism. Synthesized SMs are immediately subject to hydrolysis by sphingomyelinase (SMase), yielding ceramides, which have been shown to play a role in the development of insulin resistance and cardiovascular disorders [42, 43].

\section{Pubertal Stage Subanalysis}

In our study, pubertal stage was not associated with serum metabolite concentrations. There are, to our knowledge, no studies published which have investigated the association 
between metabolites and pubertal stage in a similar way, making a direct comparison to our results difficult. Both cross-sectional and longitudinal studies of pubertal stage indicate a decrease in insulin sensitivity during pubertal development, which is accompanied by increased fatty acid oxidation [51,52]. Further, decreased proteolysis and protein oxidation during puberty as compared to prepuberty was reported [53]. The fact that we did not observe pubertal stage-related changes in serum acylcarnitine or amino acid concentrations might indicate that these changes occur within a physiological and compensatable range, or are not captured by the metabolite panel targeted in this study.

\section{Comparison with Studies in Adults}

Data from adults and children are difficult to compare, since growth might have an impact on metabolism. Still, it is interesting to see that there is consistency as well as inconsistency concerning metabolomic changes related to obesity. Our findings of obesity-associated changes in acylcarnitine, acyl-alkyl PC, LPC and SM concentrations are largely in concordance with earlier studies in adults $[9,10,12,44,45]$. In contrast, the results concerning amino acid concentrations in our study of obese children deviate from findings in adults. For instance, branched-chain amino acids (BCAAs) and related metabolites have been consistently found increased in obese compared to lean adults [11,12,46-48], whereas we as well as Mihalik et al. [13] did not observe the same in children. In adults, one of the proposed mechanisms for the increased BCAA levels in obese and insulin-resistant subjects is reduced adipose tissue BCAA oxidation [49, 50]. Arguing in line with Mihalik et al. [13], children may exhibit early adaptive mechanisms preserving a normal BCAA metabolism in the presence of obesity and disturbed insulin sensitivity.

\section{Strengths and Limitations}

Our study has some potential limitations. Its sample size was only moderate so that the study was underpowered to detect smaller effects. Furthermore, as a cross-sectional study, it does not allow us to conclude causality from the observed differences between the weight groups. In this regard, longitudinal and interventional studies might provide further insights. Moreover, we lack information concerning diet and physical activity of the children. However, in contrast to using questionnaires, which have a very high measurement error, serum metabolite concentrations are possibly more reliable surrogates for dietary intake.

A strength of our study is that we applied a systematic metabolomics approach to compare the serum metabolome of obese children to that of normal-weight children. Furthermore, the study of metabolomics in children might lead to larger and in particular more 'true' effects than in adults, as children usually do not suffer from other diseases, take medications or smoke - factors that could influence the metabolite concentration profile [5].

In conclusion, our data provides evidence for obesity-related changes of the serum metabolite profile in children. If replicated in larger studies, the identified metabolites and metabolite ratios might be considered as potential biomarkers in the generation of new hypotheses on obesity-related pathophysiological processes.

\section{Acknowledgments}

We offer our sincere thanks to the participants of the study as well as their parents. We are grateful to Petra Nicklowitz for conducting the biochemical measurements. We thank Julia Scarpa, Katharina Sckell and Arsin Sabunchi for metabolomics measurements performed at the Helmholtz Zentrum München, Genome Analysis Center, Metabolomics Core Facility. We further thank Anja Kleinschmidt, Janina Ried and Ann-Kristin Petersen for comments, discussions, and suggestions. 
Wahl et al.: Childhood Obesity Is Associated with Changes in the Serum Metabolite Profile

\section{Funding}

This work was supported by the following grants from the German Federal Ministry of Education and Research (BMBF): grant number 01GS0820 of the National Genome Research Network, NGFNplus, grant number 01 GI0839 of the obesity competence network obesity (LARGE), grant FKZ 0315494A of the Systems Biology of Metaboty pes project, SysMBo, and grant number 03IS206IB of the Gani_Med project to WRM and the German Center for Diabetes Research (DZD e.V.). It was further supported by funding from the University of Witten/Herdecke and from the Helmholtz Zentrum München. The funders had no role in study design, data collection and analysis, decision to publish, or preparation of the manuscript.

\section{Disclosure Statement}

All authors declare that there is no conflict of interest.

\section{References}

1 Wang Y, Lobstein T: Worldwide trends in childhood overweight and obesity. Int J Pediatr Obes 2006;1: 11-25.

2 Biro FM, Wien M: Childhood obesity and adult morbidities. Am J Clin Nutr 2010;91:S1499-S1505.

3 Kiess W, Reich A, Müller G, Meyer K, Galler A, Bennek J, Kratzsch J: Clinical aspects of obesity in childhood and adolescence - diagnosis, treatment and prevention. Int J Obes Relat Metab Disord 2001;25(suppl 1):S75-79.

- 4 Lloyd LJ, Langley-Evans SC, McMullen S: Childhood obesity and adult cardiovascular disease risk: a systematic review. Int J Obes 2010;34:18-28.

5 Wang-Sattler R, Yu Y, Mittelstrass K, Lattka E, Altmaier E, Gieger C, Ladwig KH, Dahmen N, Weinberger KM, Hao P: Metabolic profiling reveals distinct variations linked to nicotine consumption in humans - first results from the KORA study. PLoS ONE 2008;3:e3863.

- 6 Vinayavekhin N, Homan EA, Saghatelian A: Exploring disease through metabolomics. ACS Chem Biol 2010; 5:91-103.

7 Calvani R, Miccheli A, Capuani G, Tomassini Miccheli A, Puccetti C, Delfini M, Iaconelli A, Nanni G, Mingrone G: Gut microbiome-derived metabolites characterize a peculiar obese urinary metabotype. Int J Obes 2010; 34:1095-1098.

- 8 Suhre K, Meisinger C, Döring A, Altmaier E, Belcredi P, Gieger C, Chang D, Milburn MV, Gall WE, Weinberger KM, Mewes HW, Hrabé de Angelis M, Wichmann HE, Kronenberg F, Adamski J, Illig T: Metabolic footprint of diabetes: a multiplatform metabolomics study in an epidemiological setting. PLoS ONE 2010;5:e13953.

9 Mihalik SJ, Goodpaster BH, Kelley DE, Chace DH, Vockley J, Toledo FGS, DeLany JP: Increased levels of plasma acylcarnitines in obesity and type 2 diabetes and identification of a marker of glucolipotoxicity. Obesity (Silver Spring) 2010;18:1695-1700.

10 Pietiläinen KH, Sysi-Aho M, Rissanen A, Seppänen-Laakso T, Yki-Järvinen H, Kaprio J, Oresic M: Acquired obesity is associated with changes in the serum lipidomic profile independent of genetic effects-a monozygotic twin study. PLoS ONE 2007;2:e218.

11 Newgard CB, An J, Bain JR, Muehlbauer MJ, Stevens RD, Lien LF, Haqq AM, Shah SH, Arlotto M, Slentz CA: A branched-chain amino acid-related metabolic signature that differentiates obese and lean humans and contributes to insulin resistance. Cell Metab 2009;9:311-326.

-12 Kim JY, Park JY, Kim OY, Ham BM, Kim H-J, Kwon DY, Jang Y, Lee JH: Metabolic profiling of plasma in overweight/obese and lean men using ultra performance liquid chromatography and Q-TOF mass spectrometry (UPLC-Q-TOF MS). J Proteome Res 2010;9:4368-4375.

13 Mihalik SJ, Michaliszyn SF, de Las Heras J, Bacha F, Lee S, Chace DH, Dejesus VR, Vockley J, Arslanian SA: Metabolomic profiling of fatty acid and amino acid metabolism in youth with obesity and type 2 diabetes: evidence for enhanced mitochondrial oxidation. Diabetes Care 2012;35:605-611.

14 Kromeyer-Hauschild K, Wabitsch M, Kunze D, Geller F, Geiß HC, Hesse V, Von Hippel A, Jaeger U, Johnsen S, Korte W: Perzentile für den Body-mass-Index für das Kindes-und Jugendalter unter Heranziehung verschiedener deutscher Stichproben. Monatsschr Kinderheilkd 2001;149:807-818.

15 Cole TJ, Bellizzi MC, Flegal KM, Dietz WH: Establishing a standard definition for child overweight and obesity worldwide: international survey. BMJ 2000;320:1240-1243.

16 Cole TJ: The LMS method for constructing normalized growth standards. Eur J Clin Nutr 1990;44:45-60.

17 Marshall WA, Tanner JM: Variations in pattern of pubertal changes in girls. Arch Dis Child 1969;44:291303. 
Wahl et al.: Childhood Obesity Is Associated with Changes in the Serum Metabolite Profile

18 Marshall WA, Tanner JM: Variations in the pattern of pubertal changes in boys. Arch Dis Child 1970;45: 13-23.

19 R Development Core Team: R: A language and environment for statistical computing. Vienna, R Foundation for Statistical Computing, 2009. www.R-project.org.

20 Mevik BH, Wehrens R: The pls package: principal component and partial least squares regression in R. J Stat Softw 2007;18:1-24.

-21 Altmaier E, Ramsay SL, Graber A, Mewes H-W, Weinberger KM, Suhre K: Bioinformatics analysis of targeted metabolomics - uncovering old and new tales of diabetic mice under medication. Endocrinology 2008;149: 3478-3489.

22 Okada T, Furuhashi N, Kuromori Y, Miyashita M, Iwata F, Harada K: Plasma palmitoleic acid content and obesity in children. Am J Clin Nutr 2005;82:747-750.

23 Houten SM, Wanders RJA: A general introduction to the biochemistry of mitochondrial fatty acid $\beta$-oxidation. J Inherit Metab Dis 2010;33:469-477.

-24 Reinehr T, Kiess W, Andler W: Insulin sensitivity indices of glucose and free fatty acid metabolism in obese children and adolescents in relation to serum lipids. Metab Clin Exp 2005;54:397-402.

25 Kim JY, Hickner RC, Cortright RL, Dohm GL, Houmard JA: Lipid oxidation is reduced in obese human skeletal muscle. Am J Physiol Endocrinol Metab 2000;279:E1039-1044.

26 Ritov VB, Menshikova EV, Azuma K, Wood R, Toledo FGS, Goodpaster BH, Ruderman NB, Kelley DE: Deficiency of electron transport chain in human skeletal muscle mitochondria in type 2 diabetes mellitus and obesity. Am J Physiol Endocrinol Metab 2010;298:E49-58.

27 Koves TR, Ussher JR, Noland RC, Slentz D, Mosedale M, Ilkayeva O, Bain J, Stevens R, Dyck JRB, Newgard CB, Lopaschuk GD, Muoio DM: Mitochondrial overload and incomplete fatty acid oxidation contribute to skeletal muscle insulin resistance. Cell Metab 2008;7:45-56.

28 Widhalm K, Zwiauer K, Hayde M, Roth E: Plasma concentrations of free amino acids during 3 weeks treatment of massively obese children with a very low calorie diet. Eur J Pediatr 1989;149:43-47.

29 Tremblay F, Lavigne C, Jacques H, Marette A: Role of dietary proteins and amino acids in the pathogenesis of insulin resistance. Annu Rev Nutr 2007;27:293-310.

30 Obici S, Wang J, Chowdury R, Feng Z, Siddhanta U, Morgan K, Rossetti L: Identification of a biochemical link between energy intake and energy expenditure. J Clin Invest 2002;109:1599-1605.

-31 Daniels MC, Ciaraldi TP, Nikoulina S, Henry RR, McClain DA: Glutamine:fructose-6-phosphate amidotransferase activity in cultured human skeletal muscle cells: relationship to glucose disposal rate in control and non-insulin-dependent diabetes mellitus subjects and regulation by glucose and insulin. J Clin Invest 1996; 97:1235-1241.

32 Patti ME, Virkamäki A, Landaker EJ, Kahn CR, Yki-Järvinen H: Activation of the hexosamine pathway by glucosamine in vivo induces insulin resistance of early postreceptor insulin signaling events in skeletal muscle. Diabetes 1999;48:1562-1571.

33 Engelmann B: Plasmalogens: targets for oxidants and major lipophilic antioxidants. Biochem Soc Trans 2004;32:147-150.

34 Kelly AS, Jacobs DR, Sinaiko AR, Moran A, Steffen LM, Steinberger J: Relation of circulating oxidized LDL to obesity and insulin resistance in children. Pediatr Diabetes 2010;11:552-555.

-35 Levitan I, Volkov S, Subbaiah PV: Oxidized LDL: diversity, patterns of recognition, and pathophysiology. Antioxid Redox Signal 2010;13:39-75.

-36 Christian AH, Mochari H, Mosca LJ: Waist circumference, body mass index, and their association with cardiometabolic and global risk. J Cardiometab Syndr 2009;4:12-19.

-37 Murugesan G, Sandhya Rani MR, Gerber CE, Mukhopadhyay C, Ransohoff RM, Chisolm GM, Kottke-Marchant $\mathrm{K}$ : Lysophosphatidylcholine regulates human microvascular endothelial cell expression of chemokines. J Mol Cell Cardiol 2003;35:1375-1384.

-38 Ma J, Folsom AR, Shahar E, Eckfeldt JH: Plasma fatty acid composition as an indicator of habitual dietary fat intake in middle-aged adults. The Atherosclerosis Risk in Communities (ARIC) Study Investigators. Am J Clin Nutr 1995;62:564-571.

-39 Hodge AM, Simpson JA, Gibson RA, Sinclair AJ, Makrides M, O’Dea K, English DR, Giles GG: Plasma phospholipid fatty acid composition as a biomarker of habitual dietary fat intake in an ethnically diverse cohort. Nutr Metab Cardiovasc Dis 2007;17:415-426.

40 Han JC, Lawlor DA, Kimm SYS: Childhood obesity. Lancet 2010;375:1737-1748.

41 Holland WL, Summers SA: Sphingolipids, insulin resistance, and metabolic disease: new insights from in vivo manipulation of sphingolipid metabolism. Endocr Rev 2008;29:381-402.

42 Hannun YA, Obeid LM: The ceramide-centric universe of lipid-mediated cell regulation: stress encounters of the lipid kind. J Biol Chem 2002;277:25847-25850.

43 Jiang XC, Paultre F, Pearson TA, Reed RG, Francis CK, Lin M, Berglund L, Tall AR: Plasma sphingomyelin level as a risk factor for coronary artery disease. Arterioscler Thromb Vasc Biol 2000;20:2614-2618.

44 Nelson JC, Jiang X-C, Tabas I, Tall A, Shea S: Plasma sphingomyelin and subclinical atherosclerosis: findings from the multi-ethnic study of atherosclerosis. Am J Epidemiol 2006;163:903-912.

-45 Younsi M, Quilliot D, Al-Makdissy N, Delbachian I, Drouin P, Donner M, Ziegler O: Erythrocyte membrane phospholipid composition is related to hyperinsulinemia in obese nondiabetic women: effects of weight loss. Metab Clin Exp 2002;51:1261-1268. 
-46 Felig P, Wahren J, Hendler R, Brundin T: Splanchnic glucose and amino acid metabolism in obesity. J Clin Invest 1974;53:582-590.

47 Caballero B, Wurtman RJ: Differential effects of insulin resistance on leucine and glucose kinetics in obesity. Metab Clin Exp 1991;40:51-58.

$\checkmark 48$ Chevalier S, Marliss EB, Morais JA, Lamarche M, Gougeon R: Whole-body protein anabolic response is resistant to the action of insulin in obese women. Am J Clin Nutr 2005;82:355-365.

-49 Pietiläinen KH, Naukkarinen J, Rissanen A, Saharinen J, Ellonen P, Keränen H, Suomalainen A, Götz A, Suortti T, Yki-Järvinen H, Oresic M, Kaprio J, Peltonen L: Global transcript profiles of fat in monozygotic twins discordant for BMI: pathways behind acquired obesity. PLoS Med 2008;5:e51.

50 She P, Van Horn C, Reid T, Hutson SM, Cooney RN, Lynch CJ: Obesity-related elevations in plasma leucine are associated with alterations in enzymes involved in branched-chain amino acid metabolism. Am J Physiol Endocrinol Metab 2007;293:E1552-1563.

51 Roemmich JN, Clark PA, Lusk M, Friel A, Weltman A, Epstein LH, Rogol AD: Pubertal alterations in growth and body composition. VI. Pubertal insulin resistance: relation to adiposity, body fat distribution and hormone release. Int J Obes Relat Metab Disord 2002;26:701-709.

52 Hannon TS, Janosky J, Arslanian SA: Longitudinal study of physiologic insulin resistance and metabolic changes of puberty. Pediatr Res 2006;60:759-763.

53 Arslanian SA, Kalhan SC: Protein turnover during puberty in normal children. Am J Physiol 1996; 270:E79-84. 